Research Article

\title{
The Association of Thyroid Nodules with Metabolic Status: A Cross-Sectional SPECT-China Study
}

\author{
Yi Chen, Chunfang Zhu, Yingchao Chen, Ningjian Wang $\mathbb{D}$, Qin Li, Bing Han, Li Zhao, \\ Chi Chen, Hualing Zhai, and Yingli Lu iD
}

Institute and Department of Endocrinology and Metabolism, Shanghai Ninth People's Hospital, Shanghai Jiao Tong University School
of Medicine, Shanghai, China

Correspondence should be addressed to Yingli Lu; luyingli2008@126.com

Received 6 December 2017; Accepted 8 February 2018; Published 6 March 2018

Academic Editor: Claudio Casella

Copyright (C) 2018 Yi Chen et al. This is an open access article distributed under the Creative Commons Attribution License, which permits unrestricted use, distribution, and reproduction in any medium, provided the original work is properly cited.

\begin{abstract}
Purpose. The aim of this study was to investigate the association of thyroid nodules (TNs) and their ultrasound (US) characteristics related to malignancy with metabolic status. Methods. The data were obtained from a cross-sectional study (SPECT-China, 20142015). The study included 9898 participants older than 18 years. Participants underwent several checkups, which included the measurement of anthropometric parameters, blood pressure, TSH levels, glucose, and lipid profiles. TN and nonalcoholic fatty liver disease (NAFLD) were diagnosed by US. TN US characteristics, including microcalcification and a taller-than-wide shape, were recorded. Results. Participants with TN $[\mathrm{TN}(+)]$ had a higher prevalence of metabolic syndrome (Met-S), obesity, central obesity, hyperlipidaemia, diabetes, hypertension, and NAFLD, especially women (all $P \leq 0.001$ ). After full adjustment, logistic regression analysis indicated that metabolic syndrome, obesity, central obesity, and hyperlipidaemia were all independent risk factors for the increased prevalence of TN in both genders $(P<0.05)$. In terms of TN US imaging characteristics associated with malignancy, being female with obesity, central obesity, and NAFLD had 1.91-fold, 2.09-fold, and 1.75-fold increased risks of developing a taller-than-wide nodule $(P=0.014,0.004$, and 0.027, resp.). Conclusions. The status of metabolic disorders might be associated with higher risks of TN in both genders. In women, obesity, central obesity, and NAFLD might contribute to the development of a taller-than-wide nodule. The potential role of metabolic status in the pathogenesis of the thyroid nodule and thyroid cancer remains to be elucidated.
\end{abstract}

\section{Introduction}

The American Thyroid Association Guidelines Task Force on Thyroid Nodules and Differentiated Thyroid Cancer [1] recently declared that thyroid nodules (TNs) are a common clinical problem, and differentiated thyroid cancer is becoming increasingly prevalent. Currently, high-resolution ultrasound (US) can detect TN in 19\%-68\% of randomly selected individuals in a population [2,3]. The clinical importance of thyroid nodules is the need to exclude thyroid cancer, which occurs in $7 \%-15 \%$ of a population [4]. In China, the prevalence of thyroid nodules is high, even in healthy adults, at approximately 30 to 50\% [5-7], and thyroid cancer has become one of the ten most common cancers in the Chinese population, especially among women [8]. Similarly, one study predicted that papillary thyroid carcinoma will become the third most common cancer in women in the United States by 2019 [9]. Based on these issues, further study of the relevant risk factors for TN and thyroid cancer is required.

The American Association of Clinical Endocrinologists (AACE)/Associazione Medici Endocrinologi (AME)/ European Thyroid Association (ETA) [10] declared that TNs are more common in elderly people, women, people with iodine deficiency, and people with a history of radiation exposure. Some thyroid function factors (i.e., TSH and thyroid antibodies) might contribute to the growth and progression of TN [11], and TNs are also closely related to a greater waist circumference (WC), higher triglyceride (TG) level, HOMA-IR, and HbAlc $[12,13]$. Among the risk factors for 
thyroid cancer, in addition to age, sex, radiation exposure history, and family history, other factors $[4,14,15]$ including obesity and central obesity may contribute to its aetiology.

Currently, the association of metabolic status with TN and thyroid cancer has not been fully investigated. The metabolic mechanisms facilitating the development of TN and thyroid cancer are complex and have not been confirmed. The data used in this study were from a large investigation, the Survey on Prevalence in East China for Metabolic Diseases and Risk Factors (SPECT-China), which was performed in 2014-2015. The objective of the present study was to investigate whether different metabolic risk factors and metabolic diseases, including obesity, central obesity, hyperlipidaemia, hypertension, diabetes, metabolic syndrome (Met-S), and nonalcoholic fatty liver disease (NAFLD), were associated with TN and their US imaging characteristics related to malignancy.

\section{Materials and Methods}

2.1. Study Participants. SPECT-China $[16,17]$ is a population-based survey that assessed the prevalence of metabolic diseases and risk factors in East China. A stratified and cluster sampling method was used. From February 2014 to December 2015, this study was performed in Shanghai, Zhejiang, Jiangxi, Jiangsu, and Anhui and in 22 sites in East China. Adults aged 18 years or older who were Chinese citizens and had lived in their current residence for more than 6 months were invited to participate in our study. Those with severe communication problems, with acute illness, or who were unwilling to participate were excluded. All participants provided written informed consent before data collection. The study protocol was approved by the ethics committee of the Shanghai Ninth People's Hospital, Shanghai Jiao Tong University School of Medicine. All procedures used were in accordance with the ethical standards of the responsible committee on human experimentation (institutional and national) and with the Helsinki Declaration of 1975, as revised in $2008[18,19]$.

Our study initially enrolled 10,441 participants older than 18 years [17-19]. Participants missing thyroid ultrasound (US) information $(n=330)$ and those with a history that included thyroid surgery, thyroid diseases (including hyperthyroidism, hypothyroidism, subacute thyroiditis, and radioactive iodine treatment history) $(n=174)$, glucocorticoid treatment $(n=32)$, amiodarone treatment $(n=1)$, or hormone replacement therapy including androgen and oestrogen $(n=6)$ were excluded. Finally, 9898 subjects were included in the final analysis. Figure 1 shows the inclusion and exclusion of participants in this analysis.

When we analysed the association between $\mathrm{TN}$ and NAFLD, participants missing liver ultrasonographic results $(n=213)$ and those with a history of excessive consumption of alcohol (male $>20 \mathrm{~g} / \mathrm{d}$, female $>10 \mathrm{~g} / \mathrm{d})$ [17] $(n=598)$, schistosomal hepatic disease $(n=5)$, or self-reported viral hepatitis (including hepatitis $\mathrm{B}$ and hepatitis $\mathrm{C}$ viruses) ( $n=110)$ were excluded; finally, 8977 subjects were included in this analysis.

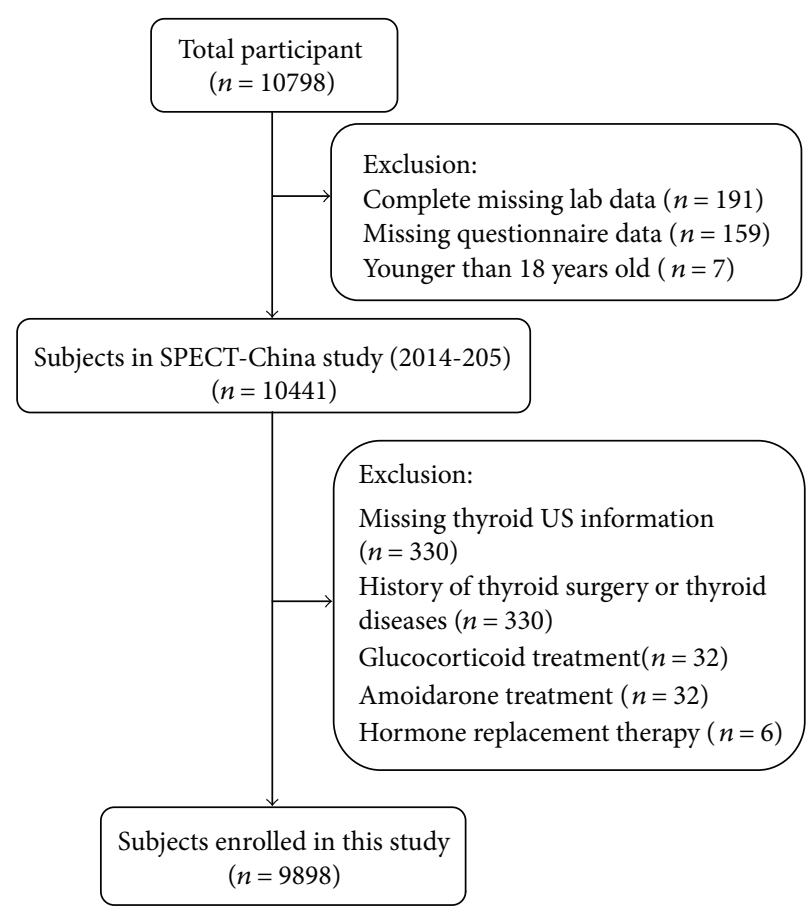

FIGURE 1: Flowchart of participants' inclusion and exclusion.

2.2. Data Collection. All data collection in this study was performed by the same staff from the Department of Endocrinology and Metabolism at the Shanghai Ninth People's Hospital, Shanghai Jiao Tong University School of Medicine. All staffs successfully completed a standard training programme that familiarized them with the specific tools and methods used. A standard questionnaire was administered by trained staff to obtain information on demographic characteristics, personal and family medical history, and risk factors in their daily lives. Weight, height, and waist and hip circumferences were measured according to a standard protocol. Blood pressure (BP) was measured on the nondominant arm 3 times consecutively with a 1-minute interval between the measurements while the participant was in a seated position after 5 minutes of rest [20]. All anthropometric measurements were conducted at the same time as the serum sample collection.

2.3. Laboratorial Assays. Serum samples for laboratorial assays were obtained by venipuncture after an 8-hour fast from 0700 to $1000 \mathrm{~h}$ in the morning. Blood samples were stored at $-20^{\circ} \mathrm{C}$ when collected and shipped by air in dry ice to one central laboratory, which was certified by the College of American Pathologists (CAP), within 2-4 hours of collection.

Fasting plasma glucose (FPG), low-density lipoprotein (LDL), high-density lipoprotein (HDL), triglycerides (TG), and total cholesterol (TC) were measured by Beckman Coulter AU 680 (Brea, USA). Insulin was detected by chemiluminescence method (Abbott i2000 SR, Chicago, USA). Glycated hemoglobin (HbAlc) was assessed by high-performance liquid chromatography (MQ-2000PT, Medconn, Shanghai, China). Thyroid peroxidase antibody 
(TPOAb), thyroglobulin antibody ( $\mathrm{TgAb})$, thyroid stimulating hormone (TSH), triiodothyronine $\left(\mathrm{T}_{3}\right)$, and thyroxine $\left(\mathrm{T}_{4}\right)$ were measured by the chemiluminescence immunoassay (Siemens, immulite 2000, Erlangen, Germany).

2.4. Thyroid and Liver Ultrasonography. Thyroid and liver US examinations were performed by the same registered physicians who were unaware of the biochemical and histological data of the participants and had professional certificates for ultrasonography awarded by the Ministry of Health of China for the use of B-mode US imaging (M7, Mindray ShenZhen, P.R. China). The characteristics of the thyroid parenchyma were described according to the echogenicity and homogeneity. Nodule characteristics, including microcalcification and taller-than-wide shape, were recorded. Hepatic steatosis was defined as a diffuse increase of the fine echoes in the liver parenchyma compared to that in the kidney or spleen parenchyma based on standard criteria [21].

2.5. Definition of Variables. Thyroid nodule: $\geq 2 \mathrm{~mm}$ in diameter. Microcalcification: calcification $<2 \mathrm{~mm}$.

Obesity was defined based upon BMI measures $\geq 28 \mathrm{~kg} /$ $\mathrm{m}^{2}$. Central obesity was defined as a waist circumferen$c e \geq 80 \mathrm{~cm}$ in women and $\geq 90 \mathrm{~cm}$ in men [20]. Based on the American Diabetes Association 2014 criteria, diabetes was defined as a previous diagnosis by healthcare professionals, fasting plasma glucose $\geq 7.0 \mathrm{mmol} / \mathrm{L}$, or $\mathrm{HbAlc} \geq 6.5 \%$. Hyperlipidaemia was defined as total cholesterol $\geq 6.22 \mathrm{~m}$ $\mathrm{mol} / \mathrm{L}$, triglycerides $\geq 2.26 \mathrm{mmol} / \mathrm{L}, \quad \mathrm{LDL}-\mathrm{C} \geq 4.14 \mathrm{mmol} / \mathrm{L}$, $\mathrm{HDL}-\mathrm{C}<1.04 \mathrm{mmol} / \mathrm{L}$, or a self-reported previous diagnosis of hyperlipidaemia by physicians. Hypertension was defined as a systolic blood pressure of $140 \mathrm{~mm} \mathrm{Hg}$ or higher, a diastolic blood pressure of $90 \mathrm{~mm} \mathrm{Hg}$ or higher, or current use of antihypertensive treatment. Metabolic syndrome (Met-S) was defined based on the International Diabetes Federation Criteria (2005). A person with MS must have abdominal obesity (waist circumference: male $\geq 90 \mathrm{~cm}$, female $\geq 80 \mathrm{~cm}$, or $\mathrm{BMI} \geq 30 \mathrm{~kg} / \mathrm{m}^{2}$ ) and any two of the following four parameters: (1) elevated $\mathrm{TG} \geq 1.7 \mathrm{mmol} / \mathrm{L}$ or treatment for dyslipidaemia; (2) reduced $\mathrm{HDL}<1.03 \mathrm{mmol} / \mathrm{L}$ in men or $<1.29 \mathrm{mmol} / \mathrm{L}$ in women or treatment for dyslipidaemia; (3) elevated blood pressure (systolic blood pressure $\geq 130$ or diastolic blood pressure $\geq 85 \mathrm{mmHg}$ ) or treatment of hypertension; and (4) elevated fasting plasma glucose $\geq 5.6 \mathrm{mmol} /$ L or a history of type 2 diabetes [22].

2.6. Statistical Analysis. We performed analyses of the survey using IBM SPSS Statistics, Version 22 (IBM Corporation, Armonk, NY, USA). All analyses were two-sided. A $P$ value $<0.05$ indicated significance. Continuous variables are presented as the mean ( \pm standard deviation) values, and categorical variables are presented as percentages. The prevalence of TN was calculated among all participants. The prevalence of nodule characteristics, including microcalcification and a taller-than-wide shape, was calculated among the participants with thyroid nodules. Continuous variables were compared using Student's $t$-test. The Mann-Whitney $U$ test was used for nonnormally distributed continuous variables, and Pearson's $\chi^{2}$ test was used for dichotomous variables. The associations of thyroid nodules and their US imaging characteristics with metabolic diseases (categorical variables) were assessed by logistic regression. The regression models were adjusted for age, smoking history (including current and past), TSH, and waist-to-hip ratio (but not including obesity, central obesity, and metabolic syndrome.). The results are expressed as odds ratios (95\% confidence interval).

Body mass index (BMI) was calculated as weight in kilograms divided by height in metre squared. Insulin resistance was estimated by the homeostatic model assessment (HOMA-IR) index: [f asting insulin $(\mathrm{mIU} / \mathrm{L})] \times[\mathrm{FPG}$ $(\mathrm{mmol} / \mathrm{L})] / 22.5$.

\section{Results}

3.1. Clinical Characteristics of Participants with and without TN. A total of 9898 subjects (4117 men; 5781 women) were enrolled in this study. The mean age was $53.34 \pm 13.07$ years, and the mean BMI was $24.52 \pm 3.52 \mathrm{~kg} / \mathrm{m}^{2}$. The prevalence of $\mathrm{TN}$ was $50.2 \%$ ( $41.3 \%$ in men and $56.5 \%$ in women). Compared to those without thyroid nodules $[\mathrm{TN}(-)]$, men with thyroid nodule(s) $[\mathrm{TN}(+)]$ were significantly older; had a significantly greater BMI, waist circumference, waist-to-hip ratio, and systolic pressure; had higher levels of FPG, HOMA-IR, HbAlc, LDL, TG, $\mathrm{T}_{4}$, TPOAb, and $\mathrm{TgAb}$; and had lower levels of HDL $(P<0.05)$. For women, significantly higher levels of TC and diastolic pressure were found as well. Furthermore, participants with TN, for both genders, had a higher prevalence of metabolic syndrome, obesity, central obesity, hyperlipidaemia, diabetes, hypertension, and NAFLD (all $P \leq 0.001$ ). The characteristics of the study subjects with and without $\mathrm{TN}$ are summarized in Table 1.

3.2. Prevalence of TN according to the Presence of Metabolic Diseases. The participants were classified into different subgroups according to whether they presented with metabolic diseases (Table 2). The prevalence of TN was significantly increased in subjects with metabolic syndrome, obesity, central obesity, hyperlipidaemia, diabetes, hypertension, and NAFLD for both genders (all $P \leq 0.001$ ).

3.3. The Relationship between TN and Metabolic Diseases. Then, the adjusted odds ratios (ORs) for TN in both genders were calculated. After adjusting for age, smoking history (including current and past), TSH, and waist-to-hip ratio (but not including obesity, central obesity, and metabolic syndrome), the logistic regression analysis indicated that metabolic syndrome (men: $\mathrm{OR}=1.31,95 \%$ CI $1.12,1.54$, $P=0.001$; women: $\mathrm{OR}=1.24,95 \%$ CI $1.09,1.43, P=0.002)$, obesity (men: $\mathrm{OR}=1.43,95 \%$ CI $1.20,1.70, P<0.001$; women: $\mathrm{OR}=1.24,95 \% \mathrm{CI} 1.05,146, P=0.013$ ), central obesity (men: $\mathrm{OR}=1.29,95 \% \mathrm{CI} 1.12,1.49, P<0.001$; women: $\mathrm{OR}=1.22,95 \% \mathrm{CI} 1.08,1.38, P=0.001)$, and hyperlipidaemia (men: $\mathrm{OR}=1.27,95 \%$ CI 1.11, 1.46, $P=0.001$; women: $\mathrm{OR}=1.14,95 \% \mathrm{CI} 1.00,1.30, P=0.045)$ were associated with $\mathrm{TN}$, whereas diabetes and NAFLD were only associated with 
TABLE 1: General characteristics of all subjects with and without thyroid nodules.

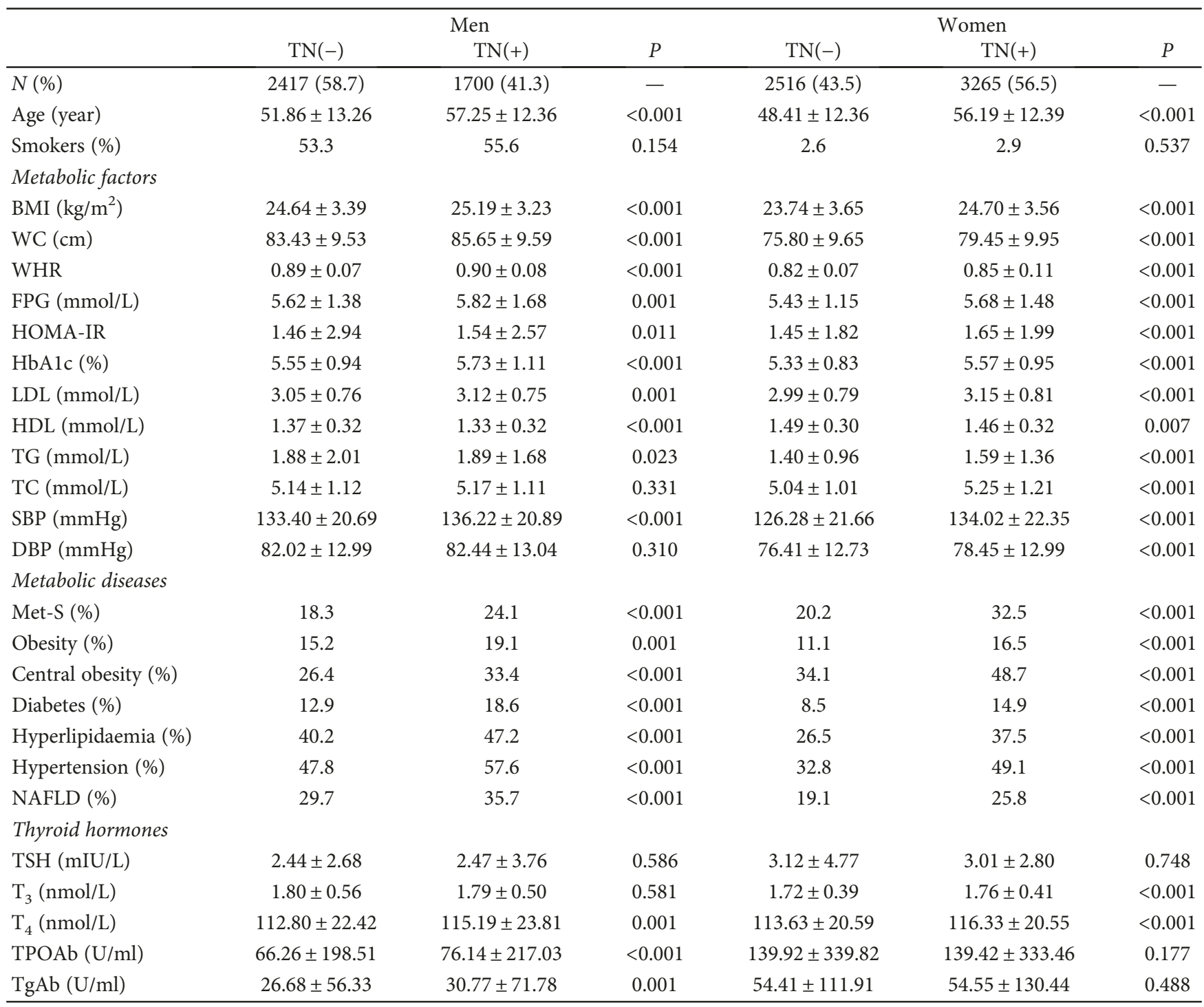

BMI: body mass index; WC: waist circumference; WHR: waist to hip ratio; FPG: fasting blood glucose; HOMA-IR: homeostasis model assessment of insulin resistance; HbAlc: glycated hemoglobin; LDL: low-density lipoprotein; HDL: high-density lipoprotein; TG: triglycerides; TC: total cholesterol; SBP: systolic blood pressure; DBP: diastolic blood pressure; Met-S: metabolic syndrome; NAFLD: nonalcoholic fatty liver disease; TSH: thyroid stimulating hormone; $\mathrm{T}_{3}$ : triiodothyronine; $\mathrm{T}_{4}$ : thyroxine; TPOAb: thyroid peroxidase antibody; TgAb: thyroglobulin antibody.

TN in men $(\mathrm{OR}=1.27,95 \% \mathrm{CI} 1.06,1.52, P=0.010$ and $\mathrm{OR}=1.43,95 \%$ CI $1.22,1.67, P<0.001$, resp.). Hypertension was not an independent significant risk factor for the increased prevalence of $\mathrm{TN}$ in both genders $(P>0.05)$ (Figure 2).

3.4. The Association of the US Imaging Characteristics of TN and Metabolic Diseases. Among the participants with TN ( $n$ $=4965$ ), the prevalence of TN with microcalcification was $1.1 \%$ ( $1.3 \%$ in men and $1.0 \%$ in women), and the prevalence of taller-than-wide-shaped TN was $2.3 \%$ (2.0\% in men and $2.5 \%$ in women).

We evaluated the relationship between the above US characteristics associated with malignancy (microcalcification and a taller-than-wide shape) with metabolic diseases (including metabolic syndrome, obesity, central obesity, hyperlipidaemia, diabetes, hypertension, and NAFLD). Among women with TN, the prevalence of taller-thanwide TN was significantly increased in participants with obesity, central obesity, and NAFLD (all $P<0.01$ ). No significant difference was found among men with TN (Table 2).

Then, we calculated the adjusted ORs for these US characteristics associated with malignancy and found that women with obesity, central obesity, and hypertension had 1.91-fold (95\% CI 1.14, 3.21, $P=0.014$ ), 2.09-fold (95\% CI 1.27, 3.46, $P=0.004$ ), and 1.75 -fold (95\% CI 1.07, 2.87, $P=0.027$ ) increased risks of developing a taller-than-wide nodule. For men, metabolic syndrome had a marginal correlation with higher risks of a nodule with microcalcification $(\mathrm{OR}=2.67$, $95 \%$ CI 1.01, 7.04, $P=0.048)$. No other significant association was detected. 
TABLE 2: Prevalence of TN and US characteristics (\%) according to the status of metabolic diseases.

\begin{tabular}{|c|c|c|c|c|c|c|}
\hline & \multicolumn{3}{|c|}{ Men } & \multicolumn{3}{|c|}{ Women } \\
\hline & $\mathrm{TN}$ & Microcalcification & Taller-than-wide shape & $\mathrm{TN}$ & Microcalcification & Taller-than-wide shape \\
\hline Met-S & 48.2 & 2.3 & 1.5 & 67.6 & 1.0 & 3.3 \\
\hline Without Met-S & 39.6 & 1.0 & 1.9 & 52.3 & 0.9 & 2.1 \\
\hline$P$ value & $<0.001$ & 0.073 & 0.596 & $<0.001$ & 0.731 & 0.102 \\
\hline Obesity & 46.9 & 1.1 & 2.8 & 66.0 & 0.8 & 4.1 \\
\hline Without obesity & 40.2 & 1.4 & 1.8 & 55.0 & 1.0 & 2.2 \\
\hline$P$ value & 0.001 & 0.646 & 0.222 & $<0.001$ & 0.696 & 0.007 \\
\hline Central obesity & 47.1 & 1.6 & 2.5 & 65.0 & 1.2 & 3.4 \\
\hline Without central obesity & 39.0 & 1.2 & 1.6 & 50.2 & 0.7 & 1.6 \\
\hline$P$ value & $<0.001$ & 0.498 & 0.207 & $<0.001$ & 0.210 & 0.003 \\
\hline Diabetes & 50.3 & 0.8 & 1.6 & 69.5 & 1.5 & 3.9 \\
\hline Without diabetes & 39.7 & 1.5 & 2.1 & 54.7 & 0.9 & 2.2 \\
\hline$P$ value & $<0.001$ & 0.371 & 0.550 & $<0.001$ & 0.318 & 0.086 \\
\hline Hyperlipidaemia & 45.3 & 1.7 & 2.5 & 64.7 & 1.2 & 2.5 \\
\hline Without hyperlipidaemia & 38.3 & 1.0 & 1.6 & 52.5 & 0.9 & 2.5 \\
\hline$P$ value & $<0.001$ & 0.276 & 0.168 & $<0.001$ & 0.361 & 0.733 \\
\hline Hypertension & 45.8 & 1.3 & 1.9 & 66.1 & 1.3 & 2.6 \\
\hline Without hypertension & 36.3 & 1.4 & 2.3 & 49.6 & 0.8 & 2.4 \\
\hline$P$ value & $<0.001$ & 0.806 & 0.576 & $<0.001$ & 0.168 & 0.572 \\
\hline NAFLD & 44.8 & 1.3 & 2.0 & 63.5 & 1.5 & 3.8 \\
\hline Without NAFLD & 38.3 & 1.0 & 2.3 & 54.0 & 0.8 & 2.0 \\
\hline$P$ value & $<0.001$ & 0.650 & 0.699 & $<0.001$ & 0.084 & 0.008 \\
\hline
\end{tabular}

Met-S: metabolic syndrome; NAFLD: nonalcoholic fatty liver disease.
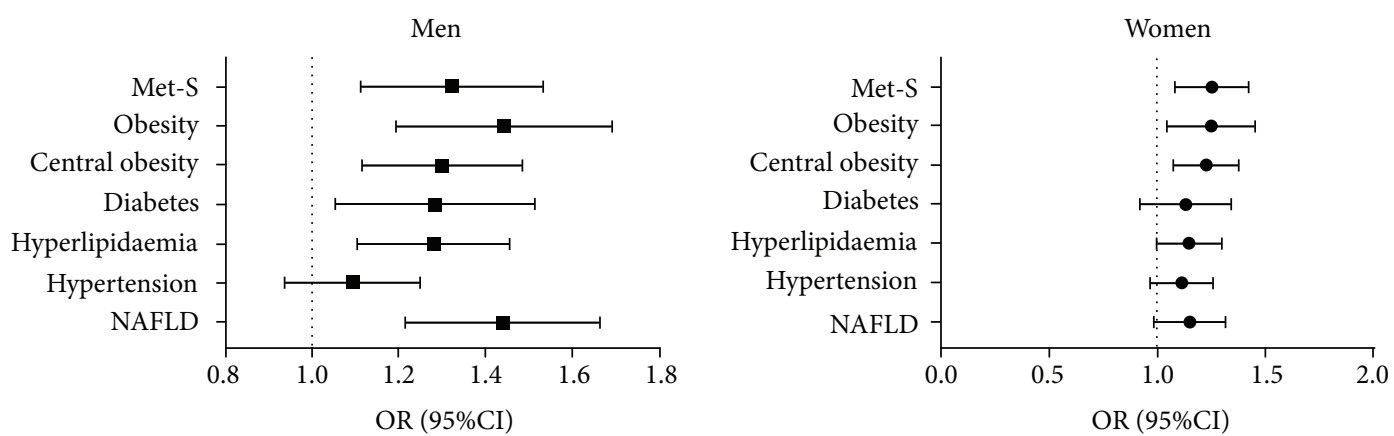

FIgURE 2: Associations of TN with metabolic diseases. The associations were analysed using logistic regression analysis. The regression models were adjusted for age, smoking history (including current and past), TSH, and waist-to-hip ratio (but not including obesity, central obesity, and metabolic syndrome).

\section{Discussion}

Thyroid US is strongly recommended as the first-line screening diagnostic test to detect thyroid lesions [23] due to its safety, noninvasive nature, and high sensitivity, and it has also been widely used to stratify the risk of malignancy in thyroid nodules and aid decision-making about whether fine needle aspiration is indicated. Researchers have consistently reported that several US grey-scale features in multivariate analyses were found to be highly likely to be malignant, including the presence of nodules with microcalcification and a taller-than-wide shape measured on a transverse view
$[1,10]$. In this study, the association of TN and their US characteristics with different metabolic diseases including obesity, central obesity, hyperlipidaemia, hypertension, diabetes, Met-S, and NAFLD was explored.

A previous study [24-27] reported that TNs are closely related to several components of Met-S. In this study, regardless of age, participants with $\mathrm{TN}$ had a significantly greater BMI, waist circumference, waist-to-hip ratio, and blood pressure as well as worse glucose and lipid profiles for both genders. Furthermore, a higher prevalence of metabolic syndrome, obesity, central obesity, hyperlipidaemia, diabetes, and hypertension was seen in the TN $(+)$ group as well. Given 
the findings that the subjects in the $\mathrm{TN}(+)$ group had worse metabolic parameters with a higher prevalence of metabolic diseases, we evaluated the adjusted ORs for TN. After full adjustment, participants of both genders with metabolic syndrome, obesity, central obesity, and hyperlipidaemia had significantly greater risks of TN, while diabetes was associated with TN in only men. To our knowledge, the literature about the relationship between NAFLD and TN remains scarce. Most recently, one published study [26] reported that the prevalence of TN in NAFLD was significantly increased in women; however, after logistic regression analyses, there was no significant difference observed. In this study, after full adjustment, the risk of TN increased by $43 \%$ in men with NAFLD. Although higher blood pressure was found in subjects with TN and the prevalence of TN was also significantly higher in participants with hypertension, there was no association between TN and hypertension after full adjustment.

In terms of thyroid cancer, currently, most studies concerning thyroid cancer and metabolic factors have focused on obesity. Several researchers have indicated that overweight and obesity are related to a modestly increased thyroid cancer risk for both genders [28-30], while similar results were found in only women in other studies [31-33]. Consistent with a previous study, in this study, the prevalence of taller-than-wide TN was significantly increased in participants with obesity and central obesity in only women. According to the logistic regression analysis, the adjusted ORs of taller-than-wide nodules were 1.91 and 2.09 in women with obesity and central obesity, respectively. Yeo et al. found that compared with nondiabetic subjects, women with preexisting DM have an increased risk of thyroid cancer [34]. Similarly, another study that focused on diabetes and thyroid cancer risk also indicated that diabetes increases the risk of differentiated thyroid cancer [35]. However, another study found the opposite result, showing that there was an inverse association between glucose and thyroid cancer risk in women [31]. In this present study, among women with $\mathrm{TN}$, the prevalence of nodules with microcalcifications or a taller-than-wide shape had no significant association with diabetes. Balkan et al. [36] reported that Met-S was not a significant risk factor of thyroid cancer following logistic regression analysis. A similar result was found in which there was no association between some metabolic factors (blood pressure, cholesterol, triglycerides, and a combined Met-S score) and thyroid cancer [31]. This study showed a 2.67 -fold increase in the risk for TN with microcalcifications among men with Met-S after full adjustment. Furthermore, women with NAFLD had a 1.75-fold increased risk of developing taller-than-wide nodules. Thus, data from this study suggested a potential role of metabolic factors besides obesity on the pathogenesis of thyroid cancer, which expanded our current understanding of the relationship between metabolism status and thyroid cancer.

Our study has several important strengths. First, we evaluated a relatively large sample of participants to examine the association of TN and their US findings related to malignancy with almost all common metabolic diseases. To our knowledge, this is the most comprehensive report to date addressing this issue. Second, all anthropometric measurements and questionnaires were completed by the same trained research group using strong quality controls. Third, we avoided a clinic-based population, and instead, communitydwelling participants living in multiple sites in China were recruited so that their results may be more representative.

However, our study also has several limitations. First, owing to the cross-sectional study nature, no causal inference can be drawn, and the reverse effect of TN and thyroid cancer causing metabolic status changes still needs to be excluded. Second, the gold standard for diagnosing a thyroid nodule, especially for thyroid cancer and NAFLD, is biopsy, and the use of ultrasonography has limitations. However, thyroid and liver biopsies may not be feasible in such a large sample. In our study, all liver and thyroid ultrasounds were performed by the same ultrasound physicians, minimizing the interobserver variability. Third, additional nodule characteristics associated with malignancy, such as hypoechoic nodules, nodules with irregular and indefinite borders, were not recorded, which needs to be further explored. Finally, selfreporting of viral hepatitis may have introduced recall bias.

\section{Conclusion}

The status of metabolic disorders might be associated with higher risks of TN in both genders. In women, obesity, central obesity, and NAFLD might contribute to the development of a taller-than-wide nodule. The potential role of metabolic status in the pathogenesis of thyroid nodules and thyroid cancer remains to be elucidated.

\section{Conflicts of Interest}

The authors declare that they have no competing interests.

\section{Authors' Contributions}

Yingli Lu designed the study. Yi Chen, Chunfang Zhu, Yingchao Chen, Ningjian Wang, Qin Li, Bing Han, Li Zhao, Chi Chen, and Hualing Zhai participated in the acquisition of data. Yingli Lu evaluated the literature. Yi Chen, Chunfang Zhu, and Yingchao Chen contributed equally to this work. Yi Chen undertook the statistical analysis and wrote the first draft of the manuscript. Chunfang Zhu and Yingchao Chen contributed to the discussion. Yingli Lu edited and revised the manuscript. All authors read and approved the final manuscript for publication. Yi Chen, Chunfang Zhu, and Yingchao Chen contributed equally to this work.

\section{Acknowledgments}

This study was supported by the National Natural Science Foundation of China (81570726); Shanghai Jiao Tong University School of Medicine (2014); Science and Technology Commission of Shanghai Municipality (16411971200); Commission of Health and Family Planning of Pudong District (PW2015D-5); the Fourth Round of Three-Year Public Health Action Plan of Shanghai by the Shanghai Municipal Commission of Health and Family Planning (15GWZK0202); Clinical Research Plan of SHDC (16CR3076B). 


\section{References}

[1] B. R. Haugen, E. K. Alexander, K. C. Bible et al., "2015 American Thyroid Association Management Guidelines for adult patients with thyroid nodules and differentiated thyroid cancer: the American Thyroid Association guidelines task force on thyroid nodules and differentiated thyroid cancer," Thyroid, vol. 26, no. 1, pp. 1-133, 2016.

[2] M. J. Yeung and J. W. Serpell, "Management of the solitary thyroid nodule," The Oncologist, vol. 13, no. 2, pp. 105-112, 2008.

[3] S. Guth, U. Theune, J. Aberle, A. Galach, and C. M. Bamberger, "Very high prevalence of thyroid nodules detected by high frequency (13 MHz) ultrasound examination," European Journal of Clinical Investigation, vol. 39, no. 8, pp. 699-706, 2009.

[4] L. Hegedus, "Clinical practice. The thyroid nodule," The New England Journal of Medicine, vol. 351, no. 17, pp. 1764-1771, 2004.

[5] W. Xu, Z. Chen, N. Li et al., "Relationship of anthropometric measurements to thyroid nodules in a Chinese population," BMJ Open, vol. 5, no. 12, article e008452, 2015.

[6] H. Jiang, Y. Tian, W. Yan et al., "The prevalence of thyroid nodules and an analysis of related lifestyle factors in Beijing communities," International Journal of Environmental Research and Public Health, vol. 13, no. 12, p. 442, 2016.

[7] Z. Chen, W. Xu, Y. Huang et al., "Associations of noniodized salt and thyroid nodule among the Chinese population: a large cross-sectional study," The American Journal of Clinical Nutrition, vol. 98, no. 3, pp. 684-692, 2013.

[8] W. Chen, R. Zheng, P. D. Baade et al., "Cancer statistics in China, 2015," CA: A Cancer Journal for Clinicians, vol. 66, no. 2, pp. 115-132, 2016.

[9] L. Davies and H. G. Welch, "Current thyroid cancer trends in the United States," JAMA Otolaryngology-Head \& Neck Surgery, vol. 140, no. 4, pp. 317-322, 2014.

[10] H. Gharib, E. Papini, R. Paschke et al., "American Association of Clinical Endocrinologists, Associazione Medici Endocrinologi, and European Thyroid Association Medical guidelines for clinical practice for the diagnosis and management of thyroid nodules: executive summary of recommendations," Endocrine Practice, vol. 16, no. 3, pp. 468-475, 2010.

[11] F. Aghini-Lombardi, L. Antonangeli, E. Martino et al., "The spectrum of thyroid disorders in an iodine-deficient community: the Pescopagano survey," The Journal of Clinical Endocrinology \& Metabolism, vol. 84, no. 2, pp. 561-566, 1999.

[12] J. Yin, C. Wang, Q. Shao et al., "Relationship between the prevalence of thyroid nodules and metabolic syndrome in the iodine-adequate area of Hangzhou, China: a cross-sectional and cohort study," International Journal of Endocrinology, vol. 2014, Article ID 675796, 7 pages, 2014.

[13] K. Wang, Y. Yang, Y. Wu et al., "The association between insulin resistance and vascularization of thyroid nodules," The Journal of Clinical Endocrinology \& Metabolism, vol. 100, no. 1, pp. 184-192, 2015.

[14] S. J. Mandel, "A 64-year-old woman with a thyroid nodule," JAMA, vol. 292, no. 21, pp. 2632-2642, 2004.

[15] A. Arduc, B. A. Dogan, M. M. Tuna et al., "Higher body mass index and larger waist circumference may be predictors of thyroid carcinoma in patients with Hürthle-cell lesion/neoplasm fine-needle aspiration diagnosis," Clinical Endocrinology, vol. 83, no. 3, pp. 405-411, 2015.
[16] N. Wang, J. Cheng, B. Han et al., "Exposure to severe famine in the prenatal or postnatal period and the development of diabetes in adulthood: an observational study," Diabetologia, vol. 60, no. 2, pp. 262-269, 2017.

[17] N. Wang, Y. Chen, Z. Ning et al., "Exposure to famine in early life and nonalcoholic fatty liver disease in adulthood," The Journal of Clinical Endocrinology \& Metabolism, vol. 101, no. 5, pp. 2218-2225, 2016.

[18] Y. Chen, Y. Chen, F. Xia et al., "A higher ratio of estradiol to testosterone is associated with autoimmune thyroid disease in males," Thyroid, vol. 27, no. 7, pp. 960-966, 2017.

[19] Y. Chen, Y. Chen, N. Wang et al., "Are thyroid nodules associated with sex-related hormones? A cross-sectional SPECTChina study," BMJ Open, vol. 7, no. 8, article e015812, 2017.

[20] Y. Xu, L. Wang, J. He et al., "Prevalence and control of diabetes in Chinese adults," JAMA, vol. 310, no. 9, pp. 948-959, 2013.

[21] H. Li, K. Dong, Q. Fang et al., "High serum level of fibroblast growth factor 21 is an independent predictor of nonalcoholic fatty liver disease: a 3-year prospective study in China," Journal of Hepatology, vol. 58, no. 3, pp. 557-563, 2013.

[22] K. G. M. M. Alberti, P. Zimmet, and J. Shaw, "The metabolic syndrome-a new worldwide definition," The Lancet, vol. 366, no. 9491, pp. 1059-1062, 2005.

[23] M. Niedziela, "Thyroid nodules," Best Practice \& Research Clinical Endocrinology \& Metabolism, vol. 28, no. 2, pp. 245277, 2014.

[24] J. H. Moon, M. K. Hyun, J. Y. Lee et al., "Prevalence of thyroid nodules and their associated clinical parameters: a large-scale, multicenter-based health checkup study," The Korean Journal of Internal Medicine, 2017.

[25] J. Shin, M. H. Kim, K. H. Yoon, M. I. Kang, B. Y. Cha, and D. J. Lim, "Relationship between metabolic syndrome and thyroid nodules in healthy Koreans," The Korean Journal of Internal Medicine, vol. 31, no. 1, pp. 98-105, 2016.

[26] X. Ding, Y. Xu, Y. Wang et al., "Gender disparity in the relationship between prevalence of thyroid nodules and metabolic syndrome components: the SHDC-CDPC community-based study," Mediators of Inflammation, vol. 2017, Article ID 8481049, 11 pages, 2017.

[27] E. Blanc, C. Ponce, D. Brodschi et al., "Association between worse metabolic control and increased thyroid volume and nodular disease in elderly adults with metabolic syndrome," Metabolic Syndrome and Related Disorders, vol. 13, no. 5, pp. 221-226, 2015.

[28] T. Pappa and M. Alevizaki, "Obesity and thyroid cancer: a clinical update," Thyroid, vol. 24, no. 2, pp. 190-199, 2014.

[29] A. G. Renehan, M. Tyson, M. Egger, R. F. Heller, and M. Zwahlen, "Body-mass index and incidence of cancer: a systematic review and meta-analysis of prospective observational studies," The Lancet, vol. 371, no. 9612, pp. 569-578, 2008.

[30] C. M. Kitahara, E. A. Platz, L. E. B. Freeman et al., "Obesity and thyroid cancer risk among U.S. men and women: a pooled analysis of five prospective studies," Cancer Epidemiology, Biomarkers \& Prevention, vol. 20, no. 3, pp. 464-472, 2011.

[31] M. Almquist, D. Johansen, T. Bjorge et al., "Metabolic factors and risk of thyroid cancer in the metabolic syndrome and cancer project (me-can)," Cancer Causes \& Control, vol. 22, no. 5, pp. 743-751, 2011.

[32] S. Rinaldi, M. Lise, F. Clavel-Chapelon et al., "Body size and risk of differentiated thyroid carcinomas: findings from the 
EPIC study," International Journal of Cancer, vol. 131, no. 6, pp. E1004-E1014, 2012.

[33] J. M. Han, T. Y. Kim, M. J. Jeon et al., "Obesity is a risk factor for thyroid cancer in a large, ultrasonographically screened population," European Journal of Endocrinology, vol. 168, no. 6 , pp. 879-886, 2013.

[34] Y. Yeo, S. H. Ma, Y. Hwang et al., "Diabetes mellitus and risk of thyroid cancer: a meta-analysis," PLoS One, vol. 9, no. 6, article e98135, 2014.

[35] B. Aschebrook-Kilfoy, M. M. Sabra, A. Brenner et al., "Diabetes and thyroid cancer risk in the National Institutes of HealthAARP diet and health study," Thyroid, vol. 21, no. 9, pp. 957-963, 2011.

[36] F. Balkan, E. D. Onal, A. Usluogullari et al., "Is there any association between insulin resistance and thyroid cancer? : a case control study," Endocrine, vol. 45, no. 1, pp. 55-60, 2014. 


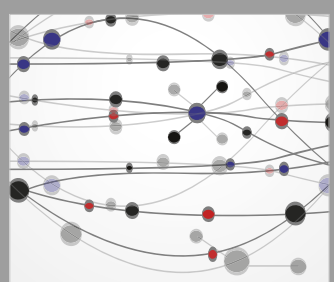

The Scientific World Journal
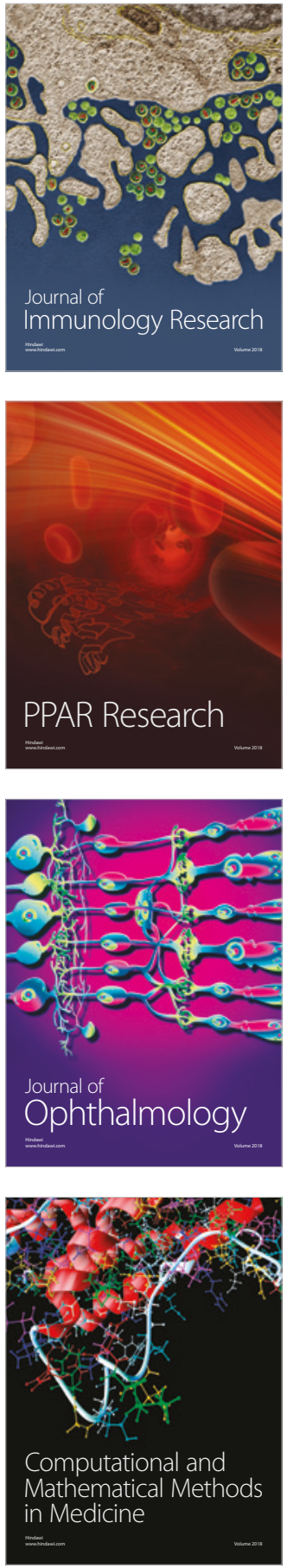

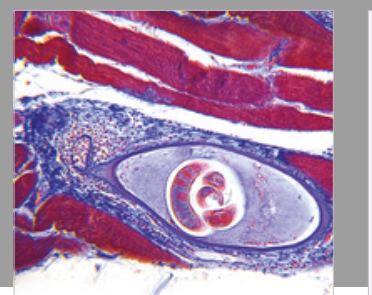

Gastroenterology Research and Practice

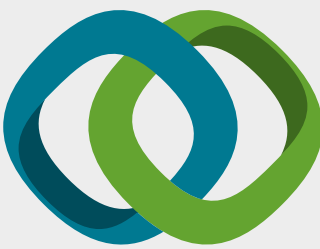

\section{Hindawi}

Submit your manuscripts at

www.hindawi.com
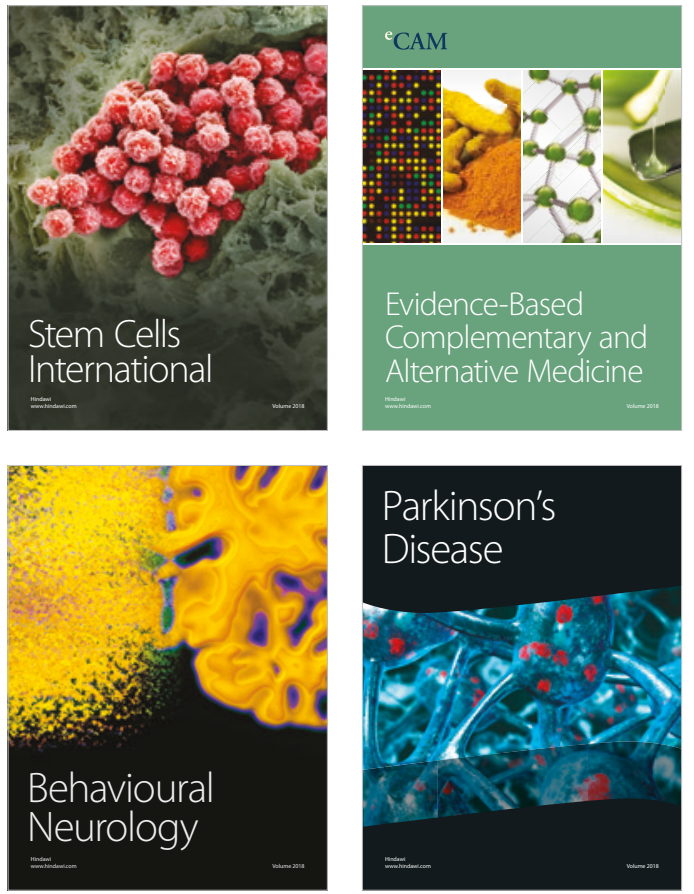

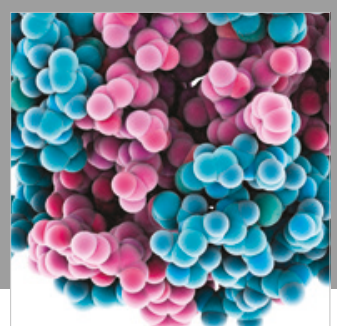

ournal of

Diabetes Research

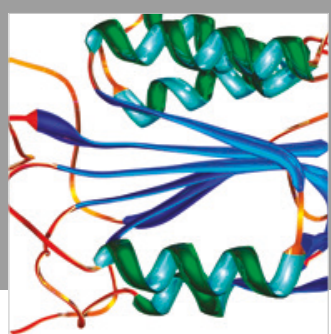

Disease Markers
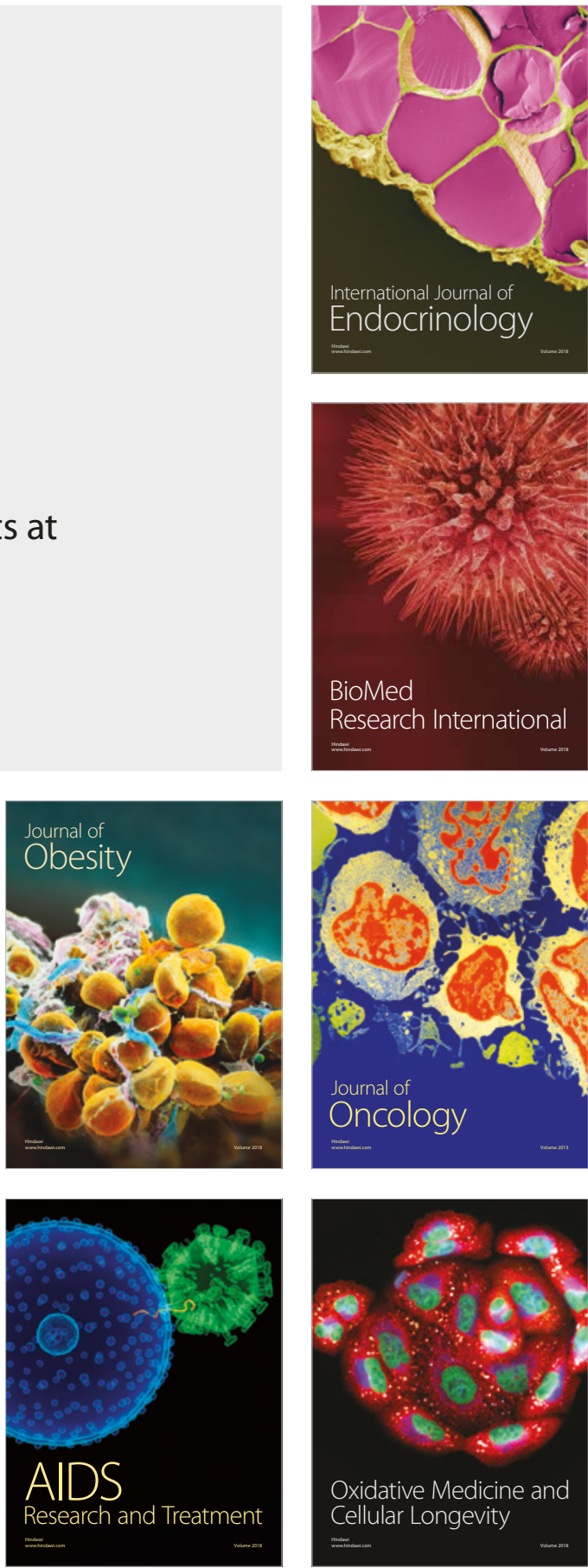